\title{
Interféromètre optique à faible cohérence couplé aux microrésonateurs optiques en polymère : nouveau protocole de biodétection
}

\section{Optical low coherence interferometer coupled to optical polymer micro-resonators: new biosensing protocol}

\author{
Yacouba SANOGO, Bernard ROUGIÉ, Martin LIÈVRE, Mohamed MALHI, Jimmy DUBARD \\ et Jean-Rémy FILTZ
}

Laboratoire commun de métrologie (LCM), Laboratoire national de métrologie et d'essais (LNE), 29 Avenue Roger Hennequin, 78197 Trappes Cedex, France, bernard.rougie@lne.fr.

\section{Résumé}

Cet article présente l'interféromètre en lumière incohérente sensible à la phase comme un nouvel outil de caractérisation de microrésonateurs pour la détection de molécules biologiques. Les mesures spatiales (interférogrammes) obtenues à l'aide de ce dispositif sont utilisées pour extraire les paramètres caractéristiques d'un microrésonateur comme son coefficient de couplage, qui permet d'étudier les différents régimes de couplage. Ce dispositif, permettant d'ailleurs d'obtenir de façon quasi simultanée les réponses en polarisation transverse magnétique et électrique, a été utilisé pour caractériser les microrésonateurs polymères en couplage vertical. Les meilleures performances ont été obtenues sur une cavité en forme d'hippodrome dans l'eau déionisée. Ce microrésonateur possède, à la longueur d'onde de $1527,7 \mathrm{~nm}$, un facteur de qualité supérieur à 38000 et une finesse de l'ordre 21. L'association du dispositif interférométrique avec un microrésonateur en polymère a été utilisée pour détecter des concentrations de glucose inférieure à $5 \mu \mathrm{g} \cdot \mathrm{mL}^{-1}$ dans l'eau déionisée en détection homogène et une densité surfacique de $4 \mathrm{ag} \cdot \mathrm{mm}^{-2}$ de TAMRA Cadavérine en détection surfacique.

MOTS CLÉS : INTERFEROMÈTRE, MICRORÉSONATEUR, MICROFLUIDIQUE, BIOCAPTEUR.

\section{Abstract}

This paper presents a low coherence interferometer as a new interrogation technique of micro-resonator for biological molecules sensing. The spatial responses obtained with PS-OLCI setup are used to evaluate relevant parameters of micro-resonator design like coupling coefficient which allows to discern different coupling configurations. This setup, which is able to provide almost simultaneously the transverse magnetic and electric responses was used to evaluate the spectral performances of micro-resonator. The best performances were obtained on a polymeric racetrack micro-resonator when immersed in deionized water. This micro-resonator displays at $1527.7 \mathrm{~nm}$ a $Q$ factor higher than 38000 and a finesse of 21. The association of the interferometric set-up and a polymer micro-resonator was allowed the detection of the concentration of glucose in deionized water lower than $5 \mu \mathrm{g} \cdot \mathrm{mL}^{-1}$ for homogeneous sensing and the surface density of TAMRA Cadaverin lower than $4 \mathrm{ag} \cdot \mathrm{mm}^{-2}$ for surface sensing.

KEY WORDS: INTERFEROMETER, MICRO-RESONATOR, MICROFLUIDIC, BIOSENSOR.

\section{Introduction}

La détection de très faibles concentrations d'espèces chimiques et biologiques représente un enjeu de plus en plus important dans des domaines comme la santé et l'environnement. En effet, la détection de molécules biochimiques, à l'aide d'outils d'analyses rapides et très sensibles, permettra, dans les domaines médical et pharmaceutique, d'effectuer des diagnostics précoces et d'augmenter les chances de guérison des patients, ou 
encore dans des domaines comme l'environnement, de détecter des polluants dans l'eau ou dans l'air.

Ces besoins croissants de biocapteurs ultrasensibles et rapides ont engendré une émergence de solutions technologiques, notamment en optique, basées sur des principes de détection très variés.

Il existe plusieurs techniques optiques de biodétection qui selon les principes utilisés peuvent être classées en deux catégories [1] : les techniques avec marquage fluorescent et celles sans marquage fluorescent.

Les techniques avec marquage fluorescent offrent les meilleures sensibilités, mais nécessitent l'utilisation d'une molécule fluorescente appelée marqueur [1]. Par ailleurs, les marqueurs fluorescents peuvent interagir avec les molécules cibles pour non seulement influencer les résultats de mesures mais aussi dénaturer la molécule étudiée.

Certes les techniques sans marquage fluorescent possèdent une moins bonne sensibilité par rapport à celles avec marquage, en revanche elles présentent l'avantage d'étudier les molécules dans leur état naturel [1].

Parmi toutes les techniques optiques de biodétection sans marqueur, les dispositifs SPR (Surface Plasmon Resonance), aujourd'hui commercialisés par plusieurs groupes internationaux notamment Biacore, sont considérés comme les plus performants avec une limite de détection de l'ordre de $1 \mathrm{pg} \cdot \mathrm{mm}^{-2}$ [1]. Les SPR sont actuellement utilisés et bien identifiés dans le domaine biomédical. Néanmoins, le coût des équipements et la nécessité de personnels qualifiés freinent encore leur apparition dans les autres secteurs tels que l'environnement.

Pour pallier cet état de fait, des recherches sont en cours dans plusieurs laboratoires afin de développer des capteurs optiques sans marqueur et bas coût capables de déceler la présence, en surface ou en solution, de molécules cibles en très faible quantité (limite de détection $<1 \mathrm{pg} \cdot \mathrm{mm}^{-2}$ ).

Dans le large éventail de capteurs étudiés, les microrésonateurs optiques, du fait de leur facteur de qualité élevé, permettent d'observer des molécules immobilisées en quantité infime sur leur surface $[1,2]$. Ce type de capteur est constitué d'une cavité résonante dans laquelle la lumière sonde se propage par réflexion totale interne, créant ainsi des ondes localisées, appelées ondes évanescentes, sur la surface du capteur. La présence de biomolécules sur la surface du capteur modifie la propagation des modes guidés par interaction entre l'onde évanescente et les molécules. Cette modification, dépendant des propriétés optiques de la molécule cible, sert à quantifier les molécules présentes en surface. Ces biocapteurs à base de microrésonateurs optiques peuvent fournir deux méthodes de détection : détection homogène et détection surfacique.

La détection homogène consiste à mesurer la variation de l'indice de réfraction optique d'une solution aqueuse dans laquelle sont réparties, de façon homogène, les molécules cibles. Cette méthode de détection a été utilisée par diverses équipes pour démontrer la capacité des microrésonateurs à détecter de faibles concentrations de biomolécules [1,2]. Cependant, cette méthode est limitée lorsque l'on recherche la sélectivité. En effet, la modification des modes guidés, liée à la variation de l'indice de réfraction optique de la solution, est due à la présence de toutes les biomolécules présentes dans la solution et pas seulement à celle de la molécule cible. Pour remédier à ce problème de sélectivité, plusieurs équipes ont utilisé la détection surfacique ou spécifique.

Dans la méthode de détection spécifique, la surface du microrésonateur est fonctionnalisée, en y accrochant des récepteurs appropriés qui permettent d'immobiliser par des liaisons covalentes les molécules cibles sur la surface. Ainsi, les molécules immobilisées et celles réparties dans la solution pourront modifier l'indice effectif du mode guidé. L'immobilisation par liaison covalente étant irréversible, la surface du capteur peut être rincée pour éliminer l'influence de la détection homogène (molécules réparties dans la solution), rendant le capteur plus sélectif. Des expériences de détections spécifiques réalisées dans divers laboratoires ont démontré les performances des microrésonateurs dans ce domaine [1,2].

Le changement de concentration de biomolécules induit une variation de l'indice du milieu entrainant la modification de la propagation des modes guidés. Cette dernière est détectée par la mesure du décalage des pics de résonnance de la réponse spectrale du microrésonateur et elle peut aussi être détectée par le changement de phase de la réponse. Cependant, les techniques optiques (balayage spectral et variation de l'intensité) utilisées à ce jour pour les interroger n'exploitent qu'une partie du signal optique propagé dans la cavité, à savoir l'amplitude de la transmission spectrale [1,2]. La sensibilité de la phase n'étant pas à ce jour utilisée, nous proposons, en conséquence, d'employer un interféromètre optique à faible cohérence sensible à la phase $[3,4]$ (Phase Sensitive-Optical Low Coherence Interferometer, PS-OLCI) comme nouveau protocole d'interrogation des microrésonateurs optiques. Ce dispositif permet de mesurer les interférogrammes exploités pour extraire les pertes de propagation, l'indice de groupe et les réponses spectrales complexes d'un composant photonique. La mesure de la phase permet non seulement d'étudier les phénomènes liés à la dispersion dans un guide d'onde, mais représente également une véritable grandeur de comparaison des performances des méthodes traditionnelles d'interrogation.

Afin de profiter simultanément des avantages des capteurs à base de microrésonateurs optiques et ceux du dispositif d'interrogation PS-OLCI, nous avons utilisé leur association comme un nouveau protocole de biodétection sans marqueur.

Nous présentons ici une brève description de la procédure de fabrication des microrésonateurs puis celle du dispositif de leur caractérisation. Les résultats 
démontrant le potentiel et l'efficacité de l'association des microrésonateurs et de l'interféromètre PS-OLCI dans le domaine de la biodétection sont également exposés.

\section{Fabrication des capteurs microrésonateurs}

Les microrésonateurs peuvent être réalisés à partir de divers matériaux comme les matériaux inorganiques (silice, semi-conducteur par exemple) ou les matériaux organiques (polymères par exemple) $[1,2,5]$. Parmi ces différents matériaux, les polymères offrent des avantages de faible coût de fabrication liés à différents procédés technologiques faciles à mettre en œuvre $[2,5]$. Le choix des matériaux polymères adéquats repose non seulement sur les propriétés optiques pour un guidage optimal de la lumière mais aussi biochimiques pour la fonctionnalisation afin d'accrocher les biomolécules cibles pour la détection surfacique. La biocompatibilité de la photo-résine époxy négative SU-8 et sa capacité à être usinée par photolithographie classique $[5,6]$ nous ont conduits à les utiliser comme cœur guidant des guides d'injection et des cavités. Parmi les deux types de couplage que sont le couplage latéral et vertical [6], notre choix s'est porté sur le couplage vertical pour sa parfaite compatibilité avec la microfluidique et l'isolement total des interactions entre le guide d'injection et la solution étudiée [5] ; ce qui simplifie l'interprétation de la signature du capteur.

Nous avons ainsi réalisé des microrésonateurs en polymère en forme d'hippodrome, couplés verticalement à un guide rectiligne, constitués d'un empilement de guides rectangulaires présentés en figure 1a. La réalisation de tels composants se déroule en trois étapes principales $[5,7]$. Les guides rectilignes permettant d'injecter la lumière dans la cavité, dont le cœur est en photo-résine époxy négative SU-8, sont d'abord réalisés par photolithographie classique sur un substrat en silicium recouvert de silice. La hauteur $h$ et la largeur $w$ typiques des guides réalisés sont respectivement de $2 \mu \mathrm{m}$ et $4 \mu \mathrm{m}$. Ensuite, une couche de polymère fluoré CYTOP, d'épaisseur 2,5 $\mu \mathrm{m}$ environ, est déposée, séparant le guide rectiligne de la cavité d'une distance d'environ $200 \mathrm{~nm}$. Cette dernière est contrôlée par gravure sèche ionique réactive (RIE, Reactive-Ion Etching). Enfin, les cavités, également en SU-8, sont formées en utilisant la même technique que pour les guides rectilignes. Sur la photographie du microrésonateur en forme d'hippodrome représenté en figure $1 \mathrm{~b}$, nous observons la structure verticale du composant présentant un alignement parfait des deux guides. Le composant réalisé est par la suite intégré dans un circuit microfluidique permettant d'introduire et de contrôler la quantité de solution à étudier. Ce capot contenant un canal microfluidique fabriqué par les procédures de lithographie douce est à base d'un matériau polymère adapté à la microfluidique, le polydiméthylsiloxane (PDMS). Il est collé sur le capteur à l'aide d'une colle polymère époxy N0A84 (Norland Optical Adhesive). Afin de réduire l'influence de la température sur les mesures, le composant optofluidique est monté sur un support thermostaté composé d'une thermistance et d'un régulateur de température.

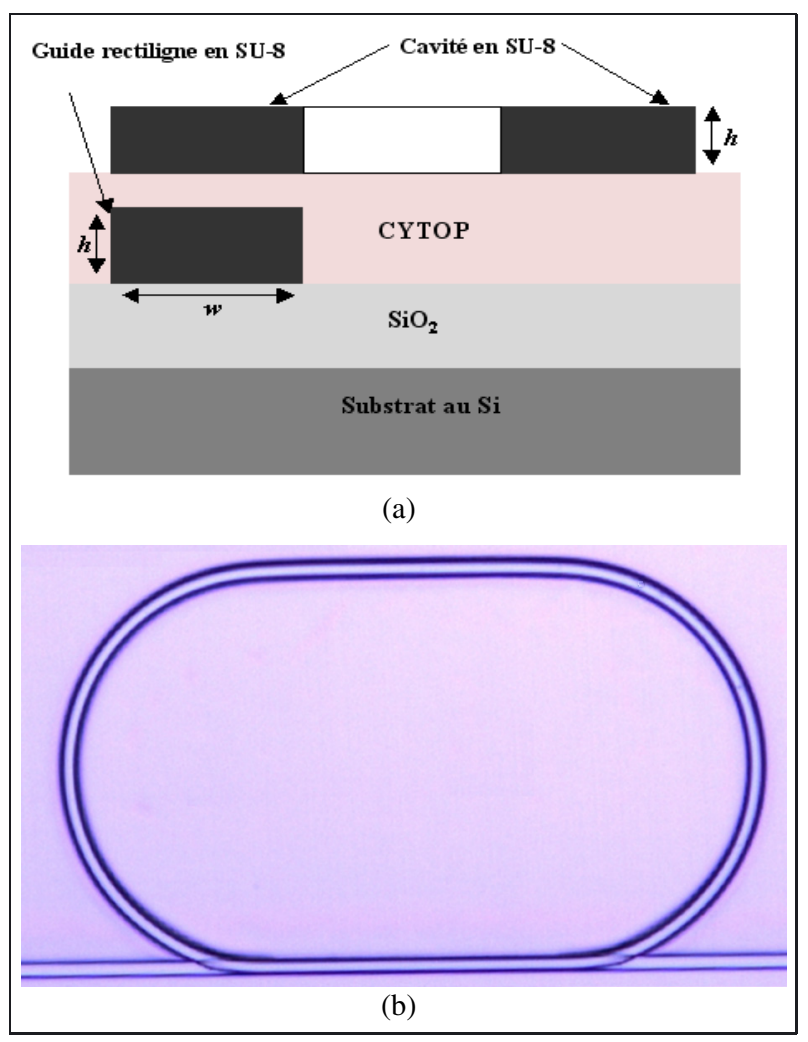

Fig. 1. - Composant optofluidique : (a) structure d'un microrésonateur en couplage vertical $(w=4 \mu \mathrm{m}$ et $h=1,8 \mu \mathrm{m})$; (b) photographie d'un microrésonateur en forme d'hippodrome en couplage vertical.

\section{Technique d'interrogation sur le principe d'un interféromètre de Michelson en lumière incohérente}

Le dispositif expérimental développé au LNE pour caractériser ces composants est un interféromètre de Michelson en lumière incohérente sensible à la phase (fig. 2) [3]. Il se compose d'une source lumineuse large bande (1 $525 \mathrm{~nm}-1605 \mathrm{~nm})$, d'un coupleur (30\%-70\%), d'un bras de test, d'un bras de référence et d'un détecteur. L'onde issue de la source est divisée en deux par le coupleur. Une partie parcourt le bras de référence et se réfléchit sur un miroir mobile, l'autre partie parcourt le bras de test et est transmise dans le composant étudié. L'onde réfléchie par le miroir mobile et celle transmise par le composant interfèrent sur le détecteur. Du fait de la faible cohérence de la source, ce signal est modulé quand la différence de marche entre le bras de référence et le bras de test est quasi nulle. Cet interféromètre est associé à un autre interféromètre de Michelson en lumière cohérente permettant d'effectuer un échantillonnage spatial (en fonction de la position du miroir) très régulier de la différence de marche.

La transformée de Fourier des données spatiales fournit des informations en amplitude dans le domaine spectral et aussi en phase. Les informations spectrales, comme le temps de groupe et la dispersion chromatique, sont obtenues à partir de la dérivée de la phase spectrale. Le temps de groupe, exploité dans la suite de cet article, 


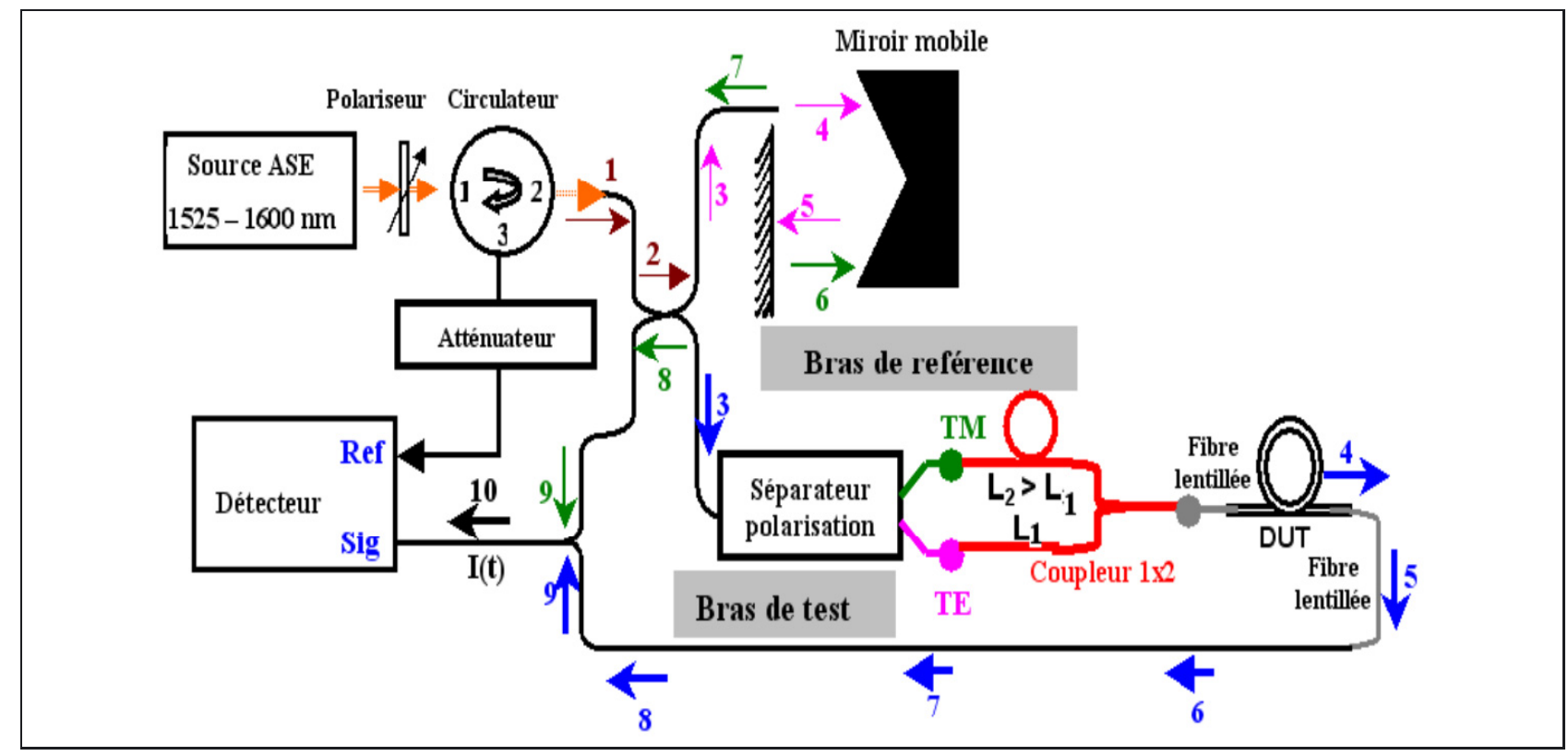

Fig. 2. - Schéma de principe du dispositif PS-OLCI.

représente la durée de propagation des composantes spectrales du signal optique dans le composant caractérisé.

Le deuxième avantage du dispositif développé réside dans le bras de test. En effet, ce bras est dédoublé par un séparateur de polarisation transverse magnétique (TM) et électrique (TE) dont les sorties sont connectées à un coupleur 50/50. Les signaux (TM) et (TE) parcourent des longueurs de fibre différentes. Cette opération, introduisant une différence de marche entre les réponses TE et TM, permet d'obtenir, en une seule mesure, les deux réponses et ainsi de réduire le temps de mesure. Pour des applications de biocapteurs, cette mesure simultanée peut être exploitée pour étudier l'orientation des molécules sur la surface du capteur.

\section{Performances du dispositif PS-OLCI}

\subsection{PS-OLCI comme outil d'aide à la conception des microrésonateurs}

Le principe de fonctionnement physique d'un microrésonateur repose sur l'interférence d'ondes multiples se propageant dans la cavité. Ainsi la réponse spatiale ou son module se caractérise par une série de pics d'interférence espacés de la circonférence optique de la cavité (fig. 3). Le premier pic observé sur la figure 3 correspond à la transmission directe dans le guide rectiligne, le deuxième à la transmission après un tour dans la cavité, le troisième après deux tours et ainsi de suite.

L'amplitude maximale de chaque pic $P_{0}, \ldots, P_{j}$ peut être exprimée en considérant la théorie des modes couplés puis la transformée de Fourier en fonction des paramètres optiques décrits ci-après $[2,8]$ :

$$
\left\{\begin{array}{l}
P_{0}=\gamma \alpha_{i}^{2}\left(1-\kappa^{2}\right) P_{i n} \\
P_{j}=\gamma \kappa^{2} a^{2 j}\left(1-\kappa^{2}\right)^{j-1} \alpha_{i}^{2(j+1)} P_{i n}
\end{array}\right.
$$

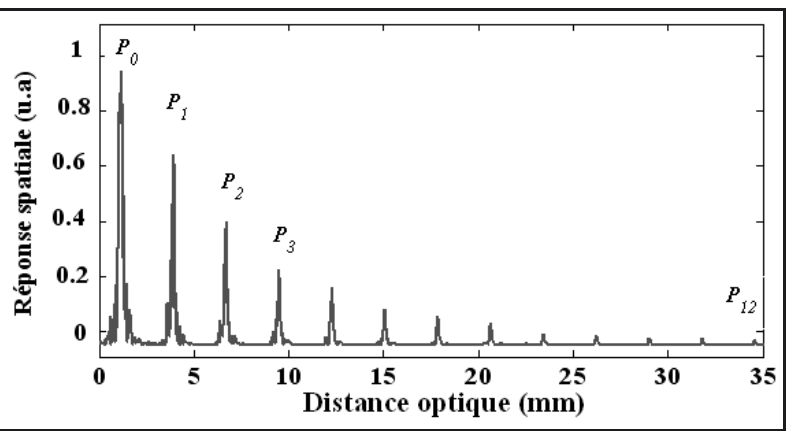

Fig. 3. - Amplitude de la réponse spatiale d'un microrésonateur en polarisation TE.

Les paramètres $a, \alpha_{i}, \kappa, \gamma$ et $P$ in représentent respectivement les pertes par tour de cavité, les pertes par couplage, le coefficient de couplage, les pertes d'insertion et l'intensité incidente. La valeur du produit $a \alpha_{i}$ et $\kappa$ peuvent directement être déterminés, à partir de (2), dès lors que la réponse spatiale est connue.

$$
\left\{\begin{array}{l}
\kappa=\sqrt{\frac{P_{1} P_{j}}{P_{1} P_{j}+P_{j+1} P_{0}}} \\
a \alpha_{i}=\sqrt{\frac{P_{j+1} P_{0}}{P_{1} P_{j}+P_{j+1} P_{0}} \frac{P_{j+1}}{P_{j}}} .
\end{array}\right.
$$

Le dispositif PS-OLCI prend d'ailleurs tout son intérêt dans la détermination de ces deux paramètres du fait qu'ils permettent de distinguer les différents régimes de couplage entre le guide rectiligne et la cavité.

En effet, pendant l'adaptation du banc PS-OLCI, initialement conçu pour caractériser les composants de télécommunication optique, nous avons caractérisé plusieurs microrésonateurs. Les réponses spatiales obtenues sur différents composants, dont l'amplitude est représentée en figure 4, symbolisent les trois régimes de couplage 


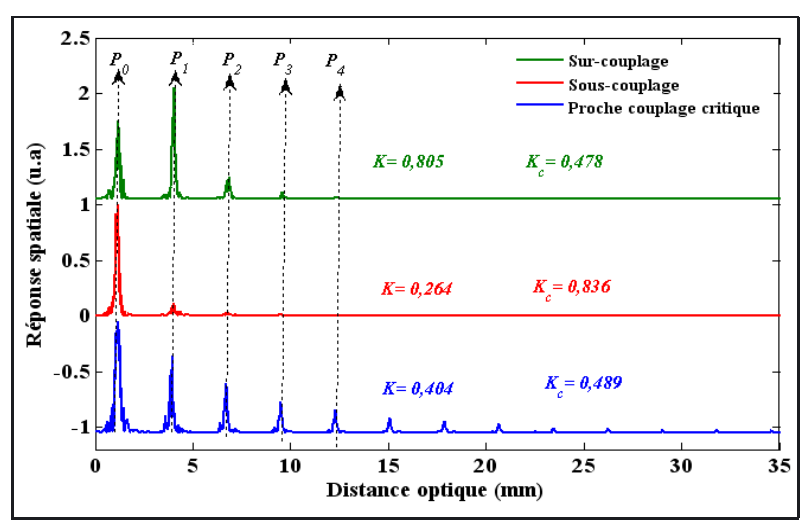

Fig. 4. - Amplitude de la réponse spatiale de microrésonateurs : distinction des différents régimes de couplage.

entre le guide rectiligne et la cavité : couplage critique, sous-couplage et sur-couplage.

Le régime de couplage critique qui permet de déduire les deux autres régimes est atteint lorsque les pertes par couplage sont égales aux pertes intrinsèques de la cavité. Le coefficient de couplage $\kappa_{c}$ associé à ce régime est défini par :

$$
\kappa_{c}=\sqrt{1-\left(a \alpha_{i}\right)^{2}} .
$$

La comparaison entre $\kappa_{c}$ et $\kappa$ est par la suite utilisée pour définir les autres régimes de couplage. Sur la première courbe (en haut) de la figure 4 , nous observons que le premier pic de l'interférogramme, correspondant à la transmission dans le guide droit est plus faible que la transmission après un tour de cavité. Cette configuration correspond au régime de sur-couplage dans lequel le coefficient de couplage est très supérieur à celui du couplage critique. La deuxième courbe (milieu), présente le cas inverse, où le coefficient de couplage est très inférieur à celui du couplage critique. C'est le régime de souscouplage. Enfin, la dernière, bien qu'elle ne corresponde pas au couplage critique (mais plus proche que les deux premiers cas), représente un régime intermédiaire qui se caractérise par une décroissance progressive des interférogrammes. Pour des applications de détection, le régime de couplage optimal est le couplage critique car il permet d'obtenir à la fois un facteur de qualité et un contraste de transmission élevés, nécessaires pour une meilleure sensibilité du capteur [9]. Les mesures PS-OLCI peuvent ainsi permettre au concepteur d'optimiser la procédure de conception et de fabrication des microrésonateurs afin de se rapprocher du régime de couplage souhaité.

\subsection{PS-OLCI comme outil de caractérisation des microrésonateurs}

Pour évaluer les performances des microrésonateurs, leurs réponses spatiales ont été mesurées en utilisant le banc PS-OLCI du LNE décrit précédemment. La figure 5 représente la réponse spatiale d'un microrésonateur en forme d'hippodrome de rayon de courbure $200 \mu \mathrm{m}$, de longueur de couplage $120 \mu \mathrm{m}$, de largeur de guide $4 \mu \mathrm{m}$ et de hauteur $2 \mu \mathrm{m}$. La première série de pics représente

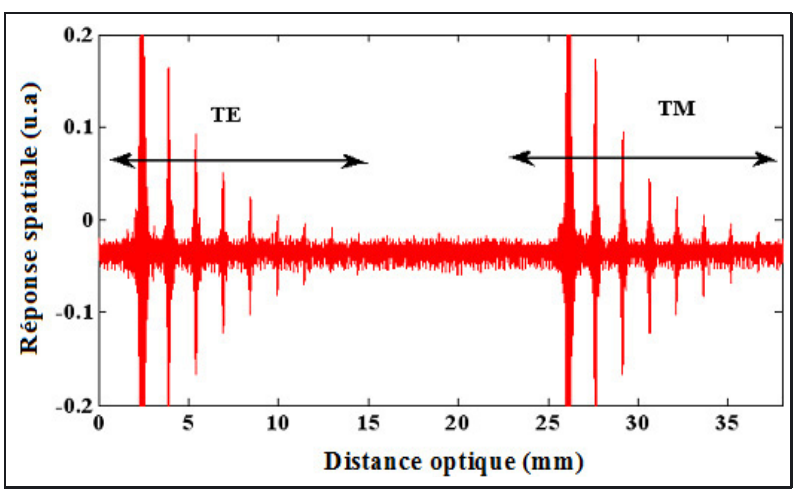

Fig. 5. - Réponse spatiale d'un microrésonateur en forme d'hippodrome en couplage vertical.
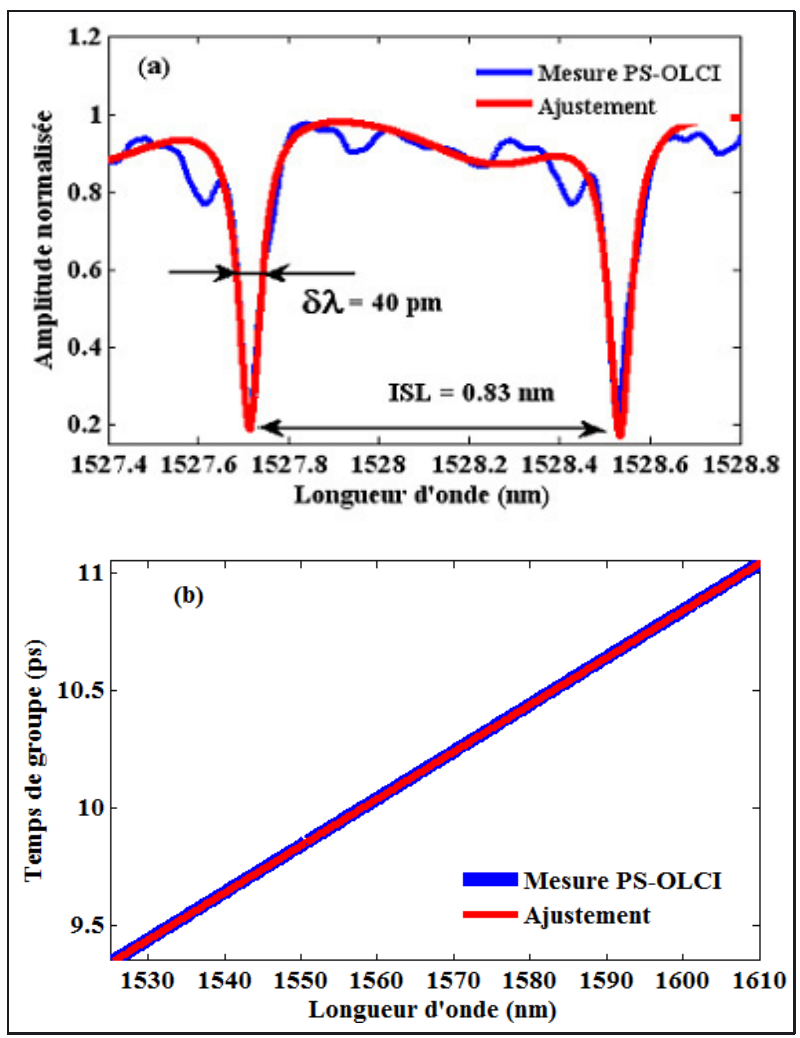

Fig. 6. - Réponse spectrale complexe en polarisation TE d'un microrésonateur en forme d'hippodrome en couplage vertical : (a) module; (b) temps de groupe déduit de la phase après un tour de cavité.

la réponse en polarisation TE et la seconde en polarisation TM. La figure 6 représente la réponse spectrale complexe (amplitude et phase) en polarisation TE obtenue en calculant la transformée de Fourier de la réponse spatiale. Les données expérimentales obtenues sur ce composant sont en très bon accord avec le calcul théorique tenant compte de la dispersion matérielle, modale et de la dispersion de polarisation. L'amplitude de la réponse spectrale, représentée sur la fenêtre spectrale $1527,4 \mathrm{~nm}-1528,8 \mathrm{~nm}$, possède à $1527,7 \mathrm{~nm}$ un intervalle spectral libre (ISL) de $0,83 \mathrm{~nm}$, une largeur à mi-hauteur (LMH) de $40 \mathrm{pm}$, une finesse de 21 et un facteur de qualité de 38 193. Ces performances sont en 


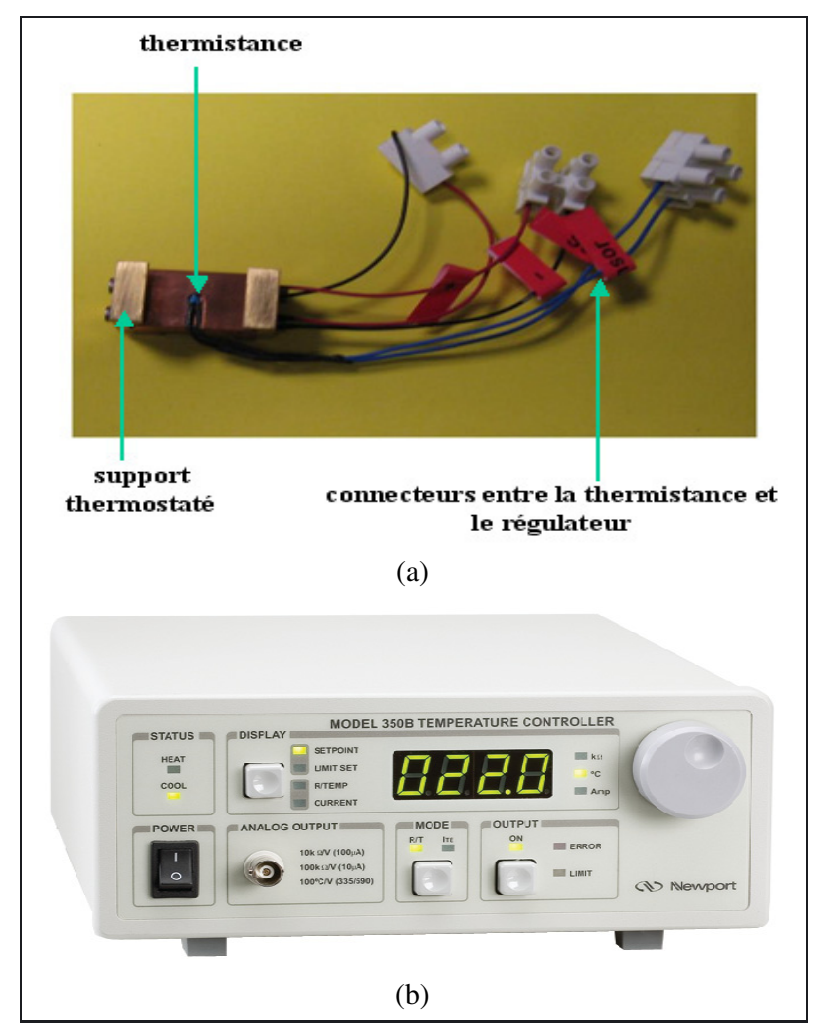

Fig. 7. - Dispositif de régulation de température : (a) photographie du support; (b) photographie du régulateur.

très bon accord avec celles obtenues à l'aide du banc de mesure en transmission [5], sur le même type de composant, par l'équipe du Laboratoire de Photonique Quantique et Moléculaire (LPQM) de l'École Normale Supérieure (ENS) de Cachan. Ceci permet ainsi de valider le dispositif PS-OLCI comme outil de caractérisation des microrésonateurs.

\section{Influence de la température sur la réponse de l'association PS-OLCI - microrésonateur}

Pour étudier l'influence de la température sur la réponse de microrésonateurs, ils ont été montés sur un support thermostaté (fig. 7), réalisé à cet effet. Ce support est composé d'une thermistance de type $\mathrm{K}$ de $10 \mathrm{k} \Omega$ (avec une tolérance de $0,88 \%$ autour de $25^{\circ} \mathrm{C}$ ) et d'un régulateur de température (modèle $350 \mathrm{~B}$ de Newport) équipé d'un PID pouvant asservir la température avec une résolution de $0,01{ }^{\circ} \mathrm{C}$.

Le microrésonateur intégré dans une cellule microfluidique a d'abord été monté sur le support thermostaté. Après l'introduction d'eau déionisée dans le canal microfluidique, à l'aide d'une seringue, nous avons mesuré la réponse du capteur en faisant varier la température par palier de $18{ }^{\circ} \mathrm{C}$ à $25^{\circ} \mathrm{C}$. Pour chaque palier, un temps de stabilisation de 10 min a été choisi, garantissant ainsi une stabilité de régulation de quelques $10^{-2}{ }^{\circ} \mathrm{C}$.

Sur la figure 8, représentant la réponse spectrale dans l'eau de ce microrésonateur, nous observons une variation de la position d'un pic de résonance et du temps de
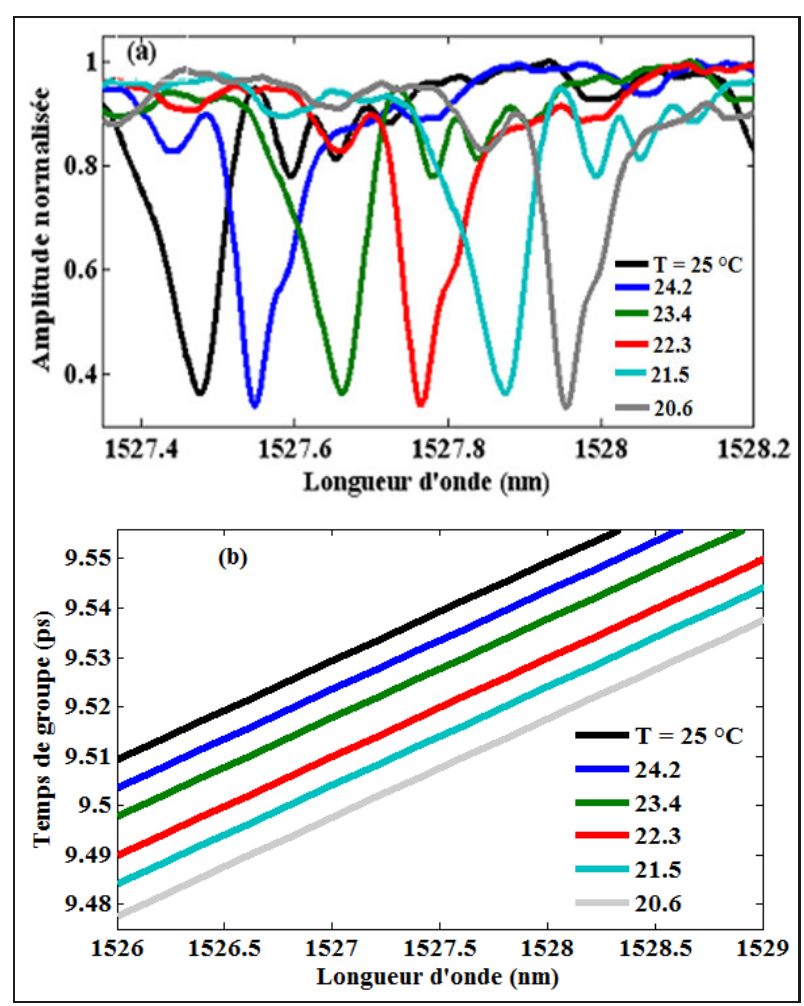

Fig. 8. - Réponse spectrale en fonction de la température : (a) module de la réponse spectrale; (b) temps de groupe (obtenu à partir de la dérivée de la phase spectrale).

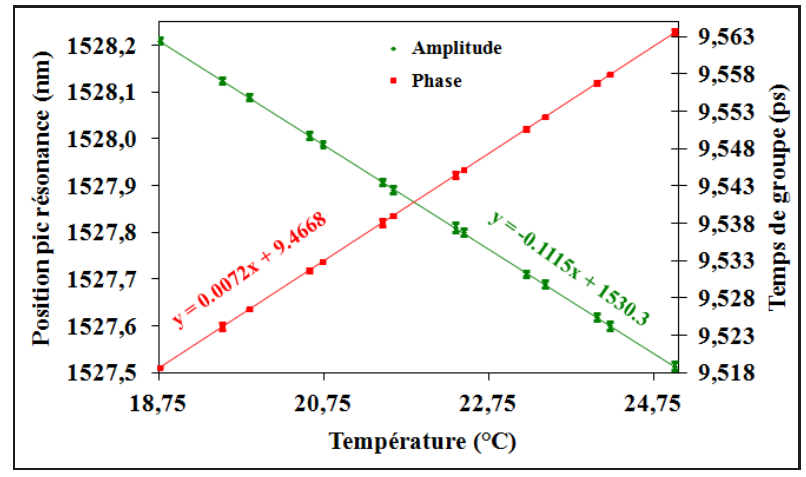

Fig. 9. - Décalage spectral et variation du temps de groupe en fonction de la température.

groupe en fonction de la température. Cette variation est liée à la dépendance de l'indice optique de l'eau à la température [10] mais aussi à la modification de l'indice de la SU-8, de la silice et du CYTOP (déformation de la structure du capteur) en fonction de la température [10]. En effet, pour des températures positives, une augmentation de la température entraîne une diminution de l'indice optique de l'eau, par conséquent une baisse de l'indice effectif du mode guidé dans le microrésonateur.

Les représentations de la position d'un pic de résonance et du temps de groupe à $1528 \mathrm{~nm}$, extraites de l'amplitude et de la phase des mesures PS-OLCI, en fonction de la température suivent des évolutions linéaires (fig. 9). La sensibilité thermique, déduite de ces 


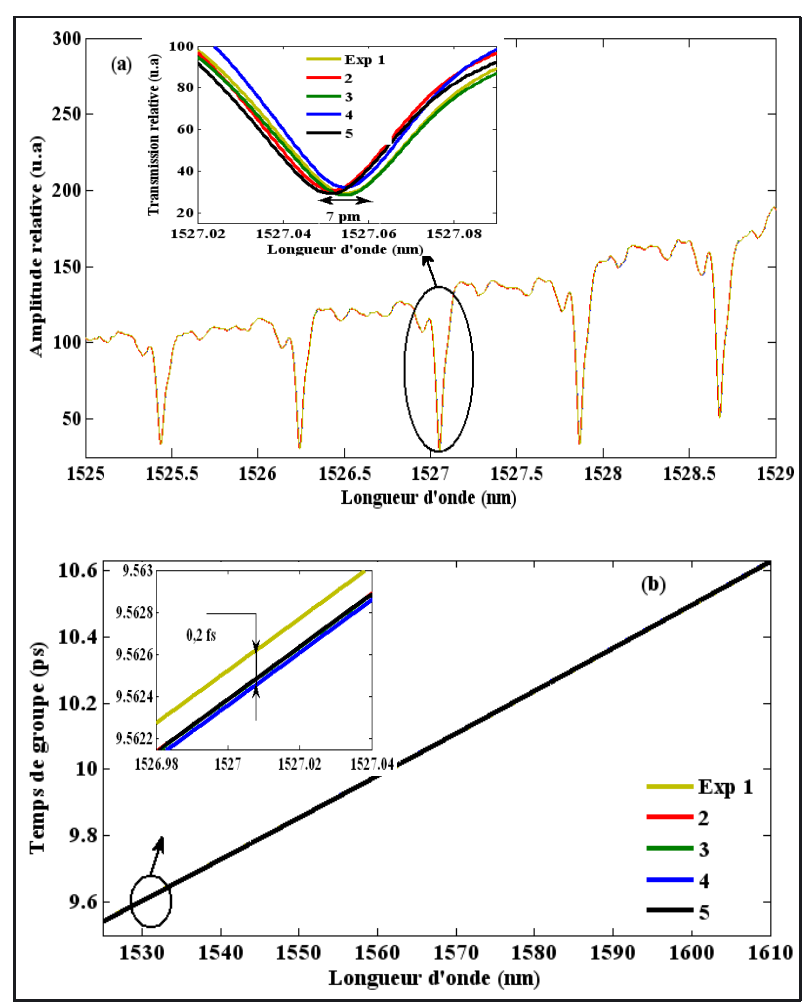

Fig. 10. - Répétabilité des mesure PS-OLCI : (a) module de la réponse spectrale; (b) temps de groupe.

données, de l'ordre de $-110 \mathrm{pm} \cdot{ }^{\circ} \mathrm{C}^{-1}$ est bien en accord avec les grandeurs publiées pour des microrésonateurs en SU-8 [11]. Celle obtenue en exploitant le temps de groupe à $1528 \mathrm{~nm}$ est de $7,2 \mathrm{fs} \cdot{ }^{\circ} \mathrm{C}^{-1}$. Le résultat de ces expériences montre la nécessité d'utiliser un dispositif thermostaté afin de maintenir le capteur à température fixe lors des expériences.

\section{Mesure de répétabilité et de reproductibilité des mesures PS-OLCI}

Avant d'entamer l'étape de détection des molécules biologiques, nous avons vérifié la répétabilité et la reproductibilité des mesures PS-OLCI à température constante. Pour cela, cinq mesures successives de la réponse du microrésonateur testé précédemment, ont été réalisées dans l'eau à $23,1{ }^{\circ} \mathrm{C}$ pour tester la répétabilité. Et cinq mesures ont été réalisées, dans les mêmes conditions, à différentes heures de la journée sur plusieurs jours, après des opérations de «montage et démontage » du composant, pour tester la reproductibilité.

Sur les figures 10 et 11 , représentant respectivement la répétabilité et la reproductibilité, nous observons, d'une acquisition à une autre, une variation de la position d'un pic de résonance et du temps de groupe. Les écarts types de répétabilité obtenus, à partir des cinq séries de mesure, sont respectivement de 7,4 pm et 0,2 fs sur la mesure de la position d'un pic de résonance et du temps de groupe. Les écarts types de reproductibilité, estimés

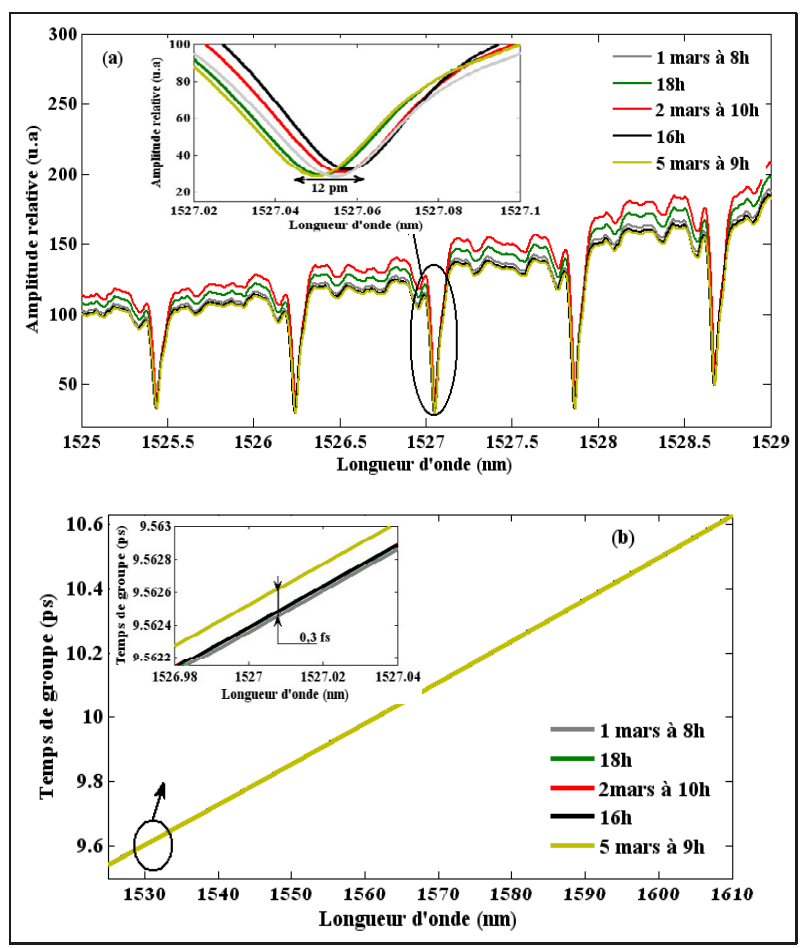

Fig. 11. - Reproductibilité des mesures PS-OLCI : (a) module de la réponse spectrale; (b) temps de groupe.

selon la norme NF ISO 5725-2 et le GUM [12], sont de $12 \mathrm{pm}$ sur le module et de 0,3 fs sur le temps de groupe.

\section{Association PS-OLCI et microrésonateur comme protocole de biodétection}

\subsection{Détection homogène}

La première expérience de biodétection a consisté à démontrer la capacité de détection homogène de la molécule de glucose. Pour cela, nous avons d'abord préparé différentes concentrations de glucose $(0 ; 0,1 ; 0,23$; $0,69 ; 1$ et 1,35$) \mathrm{mg} \cdot \mathrm{mL}^{-1}$ dans de l'eau déionisée. Ensuite, nous avons réalisé des mesures à la température de $(23,1 \pm 0,3){ }^{\circ} \mathrm{C}$, en faisant circuler les différentes solutions dans le canal microfluidique. Le passage d'une concentration plus faible à une plus forte s'effectue après avoir rincé à l'eau déionisée la surface du microrésonateur. Sur la figure 12, correspondant à la moyenne de cinq acquisitions successives par solution, nous observons un décalage spectral croissant de la position d'un pic de résonance et une variation du temps de groupe, après un tour de cavité, en fonction de la concentration. Ces variations croissantes résultent du fait qu'une augmentation de la concentration engendre une augmentation de l'indice optique de la solution, donc une croissance de l'indice effectif du mode guidé. La représentation de la valeur moyenne par solution de la longueur d'onde de résonance et du temps de groupe (après un tour de cavité), à $1530 \mathrm{~nm}$, en fonction de la concentration suit une loi linéaire (fig. 13). Les sensibilités de détection de glucose $S_{\mathrm{DG}}$, correspondant aux pentes de ces 


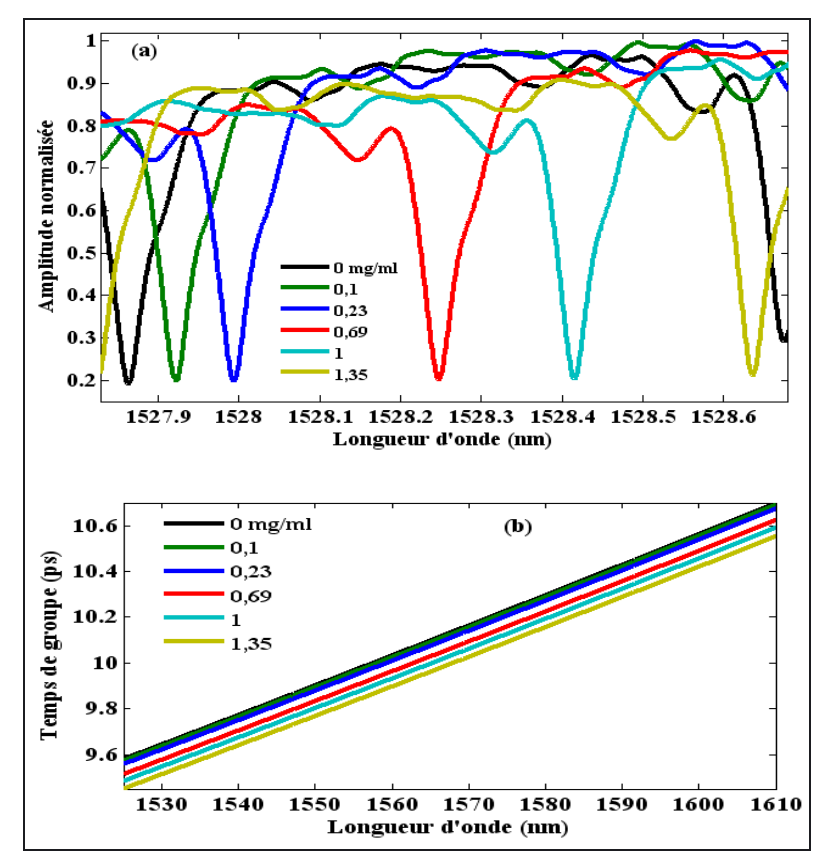

Fig. 12. - Performances spectrales en présence de glucose : (a) module de la réponse spectrale; (b) temps de groupe.

droites, obtenues en exploitant l'amplitude et la phase sont de 565,5 $\mathrm{pm} /\left(\mathrm{mg} \cdot \mathrm{mL}^{-1}\right)$ et $-24,3 \mathrm{fs} /\left(\mathrm{mg} \cdot \mathrm{mL}^{-1}\right)$, respectivement.

La sensibilité des mesures PS-OLCI, obtenue en exploitant le module, comparée à quelques résultats de détection de glucose en solution aqueuse publiés, leur est supérieure d'un facteur $10[2,13]$. Ceci est lié au fait que le facteur de qualité du microrésonateur utilisé au LNE est au moins deux fois supérieur à celui des cavités utilisées par les autres équipes citées. Ceci montre l'intérêt de réaliser des microrésonateurs possédant un haut facteur de qualité.

Afin d'évaluer l'influence des différents tours de cavité sur la sensibilité en phase, l'évolution du temps de groupe est déterminée après chaque tour de cavité en utilisant la relation suivante :

$$
T G(\lambda)=-\frac{\lambda^{2}}{2 \pi c} \frac{\partial\left(\phi_{0}(\lambda)-\phi_{j}(\lambda)\right)}{\partial \lambda}
$$

où $\lambda$ est la longueur d'onde, $\phi_{0}(\lambda)$ est la phase de la transformée de Fourier de la transmission directe dans le guide d'injection et $\phi_{j}(\lambda)$ est celle correspondant au $j^{\mathrm{e}}$ tour de cavité.

La figure 14a représente la variation du temps de groupe à $1530 \mathrm{~nm}$ en fonction de la concentration pour différents tours. Cette figure montre que cette variation qui est la sensibilité du capteur augmente avec le nombre de tours de cavité selon une relation affine, dont la pente est égale à la sensibilité après un tour $S_{1 T}$ (fig. 14b). Le nombre de tours observables, dans la cavité étant quasiment égal à sa finesse $F$, la sensibilité maximale $S_{F T}$ en phase est donc :

$$
S_{F T}=S_{1 T} F .
$$

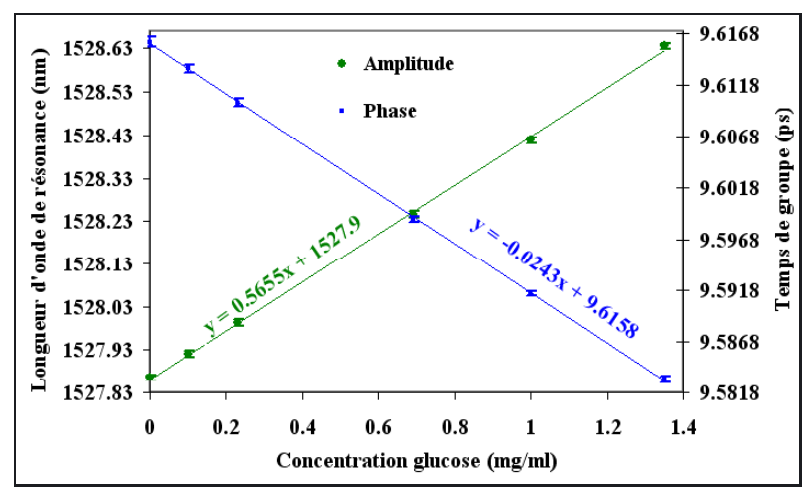

Fig. 13. - Décalage spectral et variation du temps de groupe en fonction de la concentration de glucose.

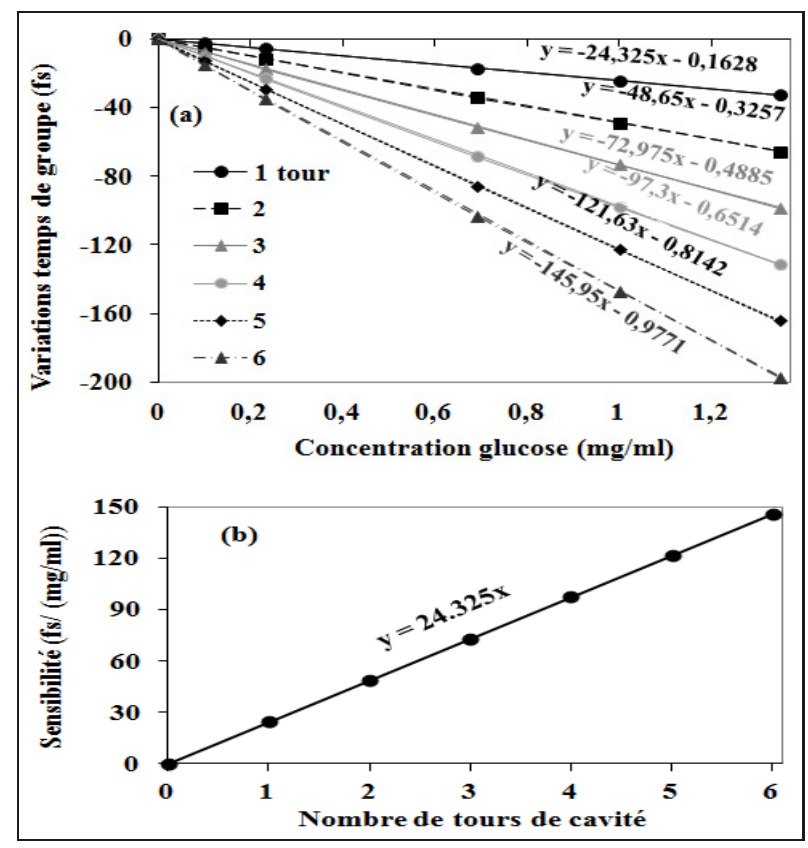

Fig. 14. - Exploitation de la phase des mesures PS-OLCI : (a) variation du temps de groupe en fonction de la concentration de glucose ; (b) variation de la sensibilité en fonction du nombre de tours de cavité.

La sensibilité totale en phase obtenue pour les expériences précédentes est de $-510,3 \mathrm{fs} /\left(\mathrm{mg} \cdot \mathrm{mL}^{-1}\right)$.

Lorsque nous considérons le double des écart types de reproductibilité des mesures PS-OLCI, comme étant le plus petit décalage détectable, nous obtenons une limite de détection, en termes de concentration de glucose, de $45 \mu \mathrm{g} \cdot \mathrm{mL}^{-1}$ sur le module et de $25 \mu \mathrm{g} \cdot \mathrm{mL}^{-1}$ sur la phase après un tour de cavité. Ces résutats montrent une limite de détection quatre fois inférieure à celle qui a été déjà publée [14]. La différence est due à l'amélioration de la valeur du facteur de qualité du microrésonnateur utilisé ici. Si nous pouvons considérer que l'incertitude sur la phase ne dépend pas du nombre de tours alors la limite de détection est de $2 \mu \mathrm{g} \cdot \mathrm{mL}^{-1}$ après $F=21$ tours. Mais si l'origine de l'incertitude sur la phase est liée à un paramètre extérieur tel que la température, ses variations 
entrainent une variation d'indice, donc de phase proportionnelle au nombre de tours. Dans ce cas la limite de détection reste inchangée avec le nombre de tours. Enfin si l'origine de l'incertitude sur la phase est liée au rapport signal à bruit alors la limite de détection est égale à celle qui est observée au tour $N$ divisée par ce nombre $N$. Pratiquement, dans l'expérience rapportée ici, l'incertitude est constante à $20 \%$ près pour les 4 à 5 premiers tours ce qui donne une limite de détection de $5 \mu \mathrm{g} \cdot \mathrm{mL}^{-1}$. Audelà l'incertitude augmente fortement. Il faut aussi noter que ce gain en limite de détection dépend de la longueur d'onde.

En comparant les résultats obtenus en exploitant la phase après $F$ tours de cavité (temps de groupe) avec ceux obtenus en exploitant l'amplitude des mesures PS-OLCI, nous remarquons que la phase présente une amélioration d'un facteur maximum égal à $F$ mais dans la pratique limité à 5. Ceci montre bien l'intérêt de la mesure de la phase, donc du dispositif PS-OLCI.

Ces premiers résultats nous ont permis de démontrer la haute sensibilité des capteurs à base de microrésonateurs optiques. Pour améliorer ces performances en apportant la sélectivité, les expériences de détection surfacique ont été réalisées.

\subsection{Démonstration de la détection surfacique}

La détection surfacique consiste à greffer sur la surface du capteur (fonctionnaliser) une substance qui va capter spécifiquement la molécule à analyser. Ce traitement apporte une sélectivité au capteur et aussi un accroissement de sa sensibilté du fait, d'une part de la proximité de la molécule et du capteur et d'autre part de l'accumulation de la molécule sur la surface. Ceci est obtenu au prix d'une complexification du capteur et aussi de la saturation de la surface à chaque utilisation, l'accrochage des molécules n'étant pas réversible.

La molécule de TAMRA cadavérine a été choisie pour caractériser le capteur dans ce type de fonctionnement. Le tétraméthyrodamine-5-carboxamide (5-TAMRA cadavérine) est une molécule organique fluorescente appartenant à la famille des amines (-NH2).

La procédure d'immobilisation et la quantification des molécules immobilisées sur la surface des microrésonateurs en SU-8 ont été mises en place par l'équipe de Malcolm Buckle du Laboratoire de Biologie et Pharmacologie Appliquée (LBPA) de l'ENS Cachan [5]. La procédure de greffage est principalement basée sur le traitement de surface au rayonnement UV sous atmosphère d'ozone pour oxyder la surface du capteur afin d'y créer des groupements fonctionnels réactifs nécessaires pour l'immobilisation des molécules cibles quantifiées par spectroscopie infrarouge.

Différentes expériences d'évaluation de la densité de molécules accrochées sur une surface de SU-8, après 20 min d'incubation de $10 \mu \mathrm{mol}$ de TAMRA cadaverine activée avec de l'EDC/NHS, ont fourni une densité

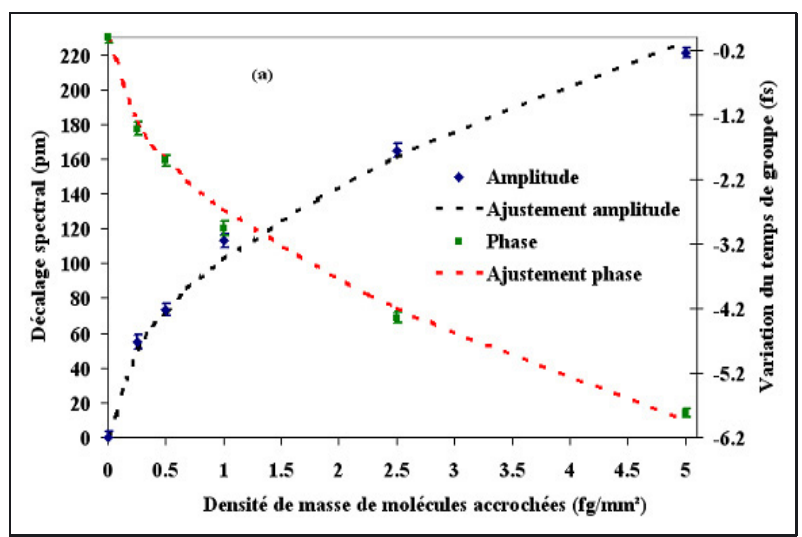

Fig. 15. - Détection de TAMRA Cadavérine : sensibilité régime statique.

de $3,2 \times 10^{9}$ molécules par $\mathrm{cm}^{2} \pm 30 \%$. Cette correspondance nous permettra de comparer nos résultats de sensibilité avec des valeurs publiées.

Des dilutions successives d'une solution de $10 \mu \mathrm{mol} \cdot \mathrm{L}^{-1}$ ont été réalisées pour obtenir des solutions de plus faible concentration $(0 ; 0,5 ; 1 ; 2 ; 5$; et 10$) \mu \mathrm{mol} \cdot \mathrm{L}^{-1}$, correspondant à des densités surfaciques de $(0 ; 0,25 ; 0,5 ; 1 ; 2$ et 5$) \mathrm{fg} \cdot \mathrm{mm}^{-2}$ (d'après les évaluations du laboratoire LBPA).

La réponse du composant est mesurée, 20 min après son immersion dans chacune des solutions précédemment réalisées. Toutes les mesures ont été effectuées à $23,1^{\circ} \mathrm{C}$.

Comme en détection homogène, sur la figure 15 , nous observons un décalage spectral croissant de la position d'un pic de résonance et une variation du temps de groupe en fonction de la densité de molécules. Ces variations croissantes sont également liées à la croissance de l'indice effectif du mode guidé avec l'épaisseur de la couche de biomolécules sur la surface du capteur. Le microrésonateur utilisé dans cette expérience n'étant pas le même que celui de la détection homogène, la mesure de répétabilité a de nouveau été effectuée. La valeur moyenne des écarts types ainsi obtenue sur la mesure de la position d'un pic de résonance (cinq acquisitions par solution) est de $15 \mathrm{pm}$. Celle obtenue sur la mesure de la pente de la phase est de 0,35 fs. Le facteur de qualité et la finesse de ce nouveau composant sont respectivement de 15000 et 14 .

La figure 15 montre une sensibilité non linéaire, pour des raisons qui tiennent pour beaucoup à la cinétique de la réaction que nous n'analyserons pas ici. La sensibilité s'accroit fortement aux faibles concentrations. Un décalage spectral de $56 \mathrm{pm}$ et une variation de temps de groupe de $-1,41$ fs correspondent à une densité surfacique de $0,25 \mathrm{fg} \cdot \mathrm{mm}^{-2}$ ou une densité volumique de $0,5 \mu \mathrm{mol} \cdot \mathrm{L}^{-1}$.

La sensibilité évaluée au voisinage de la plus faible concentration réalisée est de $290 \mathrm{~nm} /\left(\mathrm{pg} \cdot \mathrm{mm}^{-2}\right)$ et $12 \mathrm{ps} /\left(\mathrm{pg} \cdot \mathrm{mm}^{-2}\right)$, en exploitant respectivement le module et la phase après un tour de cavité des mesures PS-OLCI. 
Ce résultat est en très bon accord avec la valeur de $300 \mathrm{~nm} /\left(\mathrm{pg} \cdot \mathrm{mm}^{-2}\right)$ obtenue par Delozoide [5] sur un microrésonateur en SU-8, dont la procédure de réalisation et de fonctionnalisation est la même que le notre.

Les valeurs de sensibilité en densité molaire volumique de la solution sont de $145 \mathrm{~nm} /\left(\mu \mathrm{mol} \cdot \mathrm{L}^{-1} \cdot \mathrm{mm}^{-2}\right)$ obtenues avec le module et $6 \mathrm{ps} / /\left(\mu \mathrm{mol} \cdot \mathrm{L}^{-1} \cdot \mathrm{mm}^{-2}\right)$ obtenues avec la phase après un tour dans la cavité.

La limite de détection a été évaluée d'après les écarts types des cinq acquisitions effectuées pour chaque solution. Pour le décalage spectral du module et le décalage du temps de groupe ces écarts types sont de $15 \mathrm{pm}$ et 0,35 fs. En considérant le double de ces écarts types comme limite de détection ces valeurs limites sont de $100 \mathrm{ag} \cdot \mathrm{mm}^{-2}$ en exploitant le module et $4 \mathrm{ag} \cdot \mathrm{mm}^{-2}$ en exploitant la phase après $F$ tours (ici $F=14$ ) dans la cavité. Cette dernière valeur suppose que le rapport signal/bruit ne soit pas dégradé après 14 tours.

L'augmentation du temps d'interaction entre la molécule et le capteur peut aussi augmenter la sensibilité et réduire la limite de détection. L'analyse de la cinétique de la réaction peut aussi permettre des mesures de concentration plus précises en offrant la détermination de la non linéarité de la courbe de sensibilité observée sur la figure 15. Ces études n'ont pas été approfondies dans le temps imparti à cette étude.

Le dispositif PS-OLCI offre donc pour la détection surfacique des intérêts analogues à ceux démontrés pour la détection volumique.

\section{Conclusion et perspectives}

Nous avons présenté dans cet article, le dispositif PSOLCI comme un nouvel outil de caractérisation de microrésonateur. L'accès à la phase mais aussi la détermination des paramètres opto-géométriques étendent le nombre des paramètres mesurables d'un microrésonateur et valide l'importance de ce dispositif comme un protocole très performant d'interrogation et d'analyse des composants photoniques à base de microrésonateurs. Les premiers résultats de biodétection obtenus et présentés dans cet article ont non seulement permis de démontrer la capacité de l'association d'un PS-OLCI et d'un microrésonateur à détecter des molécules biologiques en faible concentration mais aussi de démontrer l'intérêt de l'exploitation de la phase des mesures PS-OLCI pour améliorer la limite de détection. Pour élargir ce sujet, il serait très intéressant de se pencher sur l'étude de l'orientation des biomolécules sur la surface du capteur afin de mettre à profit l'exploitation simultanée des polarisations TE et TM. Des études supplémentaires permettant d'évaluer la sensibilité du capteur à détecter des molécules présentant un groupement réactif autre que amine, comme les groupements $\mathrm{OH}, \mathrm{COOH}, \mathrm{SH}$, permettront de confirmer les potentiels de l'association PS-OLCI-microrésonateur pour des applications de biocapteurs. Enfin, l'utilisation d'une platine de translation, se déplaçant à des vitesses élevées sans introduire de vibrations parasites, à la place du moteur à courant continu du dispositif PS-OLCI permettra de suivre en temps réel la cinétique d'accrochage sur la surface du capteur afin d'en déduire les performances biologiques.

\section{Remerciements}

Ce travail a été mené à bien dans le cadre d'une étude soutenue par le Réseau National de la Métrologie Française (RNMF) fédéré par le LNE. Il a fait l'objet d'une thèse de doctorat de sciences soutenue par Yacouba Sanogo, représentant l'essentiel du projet global mené par Anne-Françoise Obaton.

\section{Références}

[1] FAN X., White I.M., SHOPOVA S.I., ZHU H., SUTER J.D. et SUN Y., "Sensitive optical biosensors for unlabeled targets: A review", Anal. Chim. Acta, 620, 2008, 8-26.

[2] CHAO C.-Y. et GUO L.J., "Polymer microring resonators for biochemical sensing applications", IEEE J. Sel. Top. Quant. Electron., 12, 1, 2006, 148-155.

[3] Obaton A.-F., Quoix A. et Dubard J., "Uncertainties on distance, and chromatic dispersion measurement using Optical Low Coherence Reflectometry", Metrologia, 45, 2008, 83-92.

[4] Obaton A.-F., Palavicini C., JaOuËn Y., KeRRINCKX E., QUIQUEMPOIS Y. et LIÈVRE M., "Characterization of fiber Bragg gratings by phase-sensitive optical low coherence reflectometry", IEEE Trans. Instrum. Meas., 55, 5, 2006, 1696-1703.

[5] Delezoide C., Salsac M., Lautru J., Leh H., Nogues C., Zyss J., Buckle M., LedouX-RAK I. et NGUYeN C.T., "Vertically coupled polymer microracetrack resonators for label-free biochemical sensors", IEEE Phot. Technol. Lett., 34, 4, 2011, 270-272.

[6] Shen B.Y., Chen Y.C. et Tsai Y.H., "Monolithic SU-8 micro-interferometer for biomedical detections", Sens. Actuators, 144, 2008, 299-306.

[7] SANOGO Y., " Conception et fabrication de capteurs et de leur technique d'interrogation pour des applications dans les domaines de la santé et de l'environnement », Thèse doctorat de sciences, ENS Cachan, France, 10 décembre 2012.

[8] YARIV A., "Universal relations for couling of optical power between microresonators and dielectric waveguides", Electron. Lett., 36, 2000, 321-322.

[9] GotTesman Y., RaO E.V.K. et RABUs D.G., "New methodology to evaluate the performance of ring resonators using optical low-coherence reflectometry", J. Lightwave Technol., 22, 6, 2004, 1566-1572.

[10] Schiebener P., Straub J., LeVelt Sengers J.M.H. et GALLAGHER J.S., "Refractive index of water and steam as function of wavelength, temperature and density", International Association for the Properties of Water and Steam, 1997. 
[11] Fabrizio G., Emiliano D., Caterina S., Lorenzo D. et Francesco M., "Experimental determination of the sensitivity of Bloch surface waves based sensors", Opt. Exp., 18, 8, 2010, 8087.

[12] Guide pour l'expression de l'incertitude de mesure/Guide to the expression of uncertainty in measurement (GUM), JCGM 100:2008, BIPM.

Article reçu le 18 avril 2014.
[13] Kim G.D., Son G.-S., Le H.-S., KIM K.D. et LeE S., "Integrated photonic glucose biosensor using a vertically coupled microring resonator in polymers", Opt. Commun., 281, 2008, 4644-4647.

[14] Obaton A.-F., SANogo Y., Yardin C., LaUtru J., LyATHAUd C., DUbard J. et Fisher N., "Association of vertically coupled polymer micro resonator and phase sensitiveoptical low cohrence interferometr for label free biosensing applications", Metrologia, 51, 1, 2014, 1-10. 